\title{
Genetic Purity Analysis in Maize under Temperate Conditions
}

\author{
M.I. Bhat ${ }^{\text {* }}$, Gulzaffar ${ }^{2}$, Z.A. Dar ${ }^{1}$, A.A. Lone ${ }^{1}$, A.B. Shikari ${ }^{1}$, G. Ali ${ }^{1}$, \\ M.A. Wani ${ }^{1}$, G.H. Khan ${ }^{1}$, A. Gazal ${ }^{1}$ and R.A. Lone ${ }^{1}$ \\ ${ }^{1}$ AICRP, NSP, SKUAST-K, Srinagar, J\&K, India \\ ${ }^{2}$ DARS, Budgam (SKUAST-K), Srinagar, J\&K, India \\ *Corresponding author
}

\section{A B S T R A C T}

Keywords

Genetic, Analysis, Temperature, SSRs.

Article Info

Accepted:

26 August 2017

Available Online:

10 September 2017
In the present study SSR markers were shown to be polymorphic between parents which would help in establishing the genetic purity of hybrids in commercial seed lots. Also, few SSRs were found to discriminate among the set of hybrids which will ease the seed identity of competing hybrids in seed chain. Low probability of identity based on marker information suggests that a set of markers identified in present study can be efficiently used in establishing distinctness, identity and purity of hybrids in open markets after their release.

\section{Introduction}

Maize (Zea mays L.) is the second most important cereal crop in the world after wheat (Asif et al.,2006) and is produced on nearly 100 million hectares in developing countries with almost $70 \%$ of the total maize production in the developing world coming from low to lower middle income countries. The demand for maize is projected to get doubled by the year 2050 and the crop may attain highest production globally by 2025 (Rosergent et al., 2009). In India the crop occupies an area of 8.55 million hectares and the production is 21.76 million tons with an average yield of 2.48 tones $^{\text {ha- }}{ }^{1}$ (Anonymous, 2013). In Jammu and Kashmir State, maize is grown as rainfed crop in marginal hilly terrains of Kashmir Valley and occupies an area of 0.1 million hectares with production and productivity of 0.15 million tones and 1.2 tones per hectare
(Anonymous, 2012). Although, number of varieties has been released for valley basin and high altitude areas of J\&K, however, few hybrids/ composites have become popular in maize growing regions and have successfully been grown by farmers with the aim of achieving high production. The maize hybrids are known for their improved yield performance, genetic uniformity, resistance to pests and diseases and are being preferred by the farmers. Thus, genetic purity of seed becomes much more important since seed is the initial lever for bolstering production potentiality and means of creating surplus in commercial markets (Srivastava and Jaffee, 1993). Hybrid corn has helped to achieve a quantum jump in yield. Before dispensing seeds to the farmer its purity testing is essential to ensure its trueness. It is estimated 
that for every percent impurity in hybrid seed the yield reduction can go upto $100 \mathrm{~kg} \mathrm{ha}^{-1}(\mathrm{Li}$ Liu et al., 2000). In maize it is even higher as the reduction in the yield goes to $135 \mathrm{~kg}$ ha ${ }^{1}$ (Mao et al., 1996). Moreover, the contamination reduces the genetic and physiological quality of seeds that consequently decreases the crop productivity (Kalinka et al., 2006). Since detailed morphological descriptors of the plants and seeds have been used for identification but this method is ambiguous and time consuming. The methods like grow out test normally demand considerable time and labour. Hybrid purity testing is best achieved by use of DNA markers. Molecular markers which could clearly distinguish the hybrids from its parental lines have been identified and developed in major agricultural and horticulture crops (Pallavi et al., 2010). SSR markers have proven to be preferred and robust molecular markers for purity identification, due to their high efficiency, reproducibility and simplicity (Wu et al., 2010). Genetic purity of commercial seed lots is of immense importance in realization of full yield potential of varieties per unit area. Therefore, present study was broadly aimed to identify the DNA based markers for hybrid purity testing.

\section{Materials and Methods}

The plant materials comprised of six maize (Zea mays L.) inbreds (KDM-361A,KDM343A,KDM-332A,KDM-914A, KDM-916A, KDM-362A,CM-128) and five single cross hybrids namely H1 (KDM-361A X KDM343A), H2 (KDM-332A X CM-128),H3 (KDM-914A X KDM-362A),H4 (KDM916A X CM-128),H5 (KDM-332A X KDM916A). A set of inbreds and hybrids was laid in Replicated Block Design with three replications. The observations were recorded for plant height, ear height, tassel length, ears per plant, days to tassel emergence, days to silk emergence, days to maturity, ear length, ear girth, number of rows per ear, number of grains per row, number of grains per ear, grain yield per plant. Analysis of variance was performed following Singh and Chowdhury (1985). Besides, total of thirty one morphological traits were studied as per Maize descriptor (IIMR, New Delhi).DNA extraction from young leaves was carried out by CTAB (Cetyl- Tri Methyl Ammonium Bromide) method as described by Murray and Thompson (1980).PCR assay was performed using 45 SSR markers retrieved from www.maizegdb.org (Table 1). PCR reaction mix contained $\sim 25 \mathrm{ng}$ of DNA, 10x PCR buffer (10 mMTris, pH 8.4, $50 \mathrm{mMKCl}, 1.8$ $\mathrm{mM} \mathrm{MgCl}_{2}$ ), $2 \mathrm{mM}$ dNTPs (MBI, Fermentas, Lithuania, USA), 5 pmol each of forward and reverse primer and $1 \mathrm{U}$ of Taq DNA polymerase (Bangalore GeneiPvt.Ltd., Bangaluru, India) in a reaction volume of 10 $\mu 1$. Polymerase chain reaction (PCR) was performed in a thermal cycler (Eppendorf, Hamburg, Germany) with following thermal regimes: After initial denaturation for $4 \mathrm{~min}$ at $94^{\circ} \mathrm{C}$, each cycle comprised $1 \mathrm{~min}$ denaturation at $94^{\circ} \mathrm{C}, 1 \mathrm{~min}$ annealing at $55^{\circ} \mathrm{C}$, and $2 \mathrm{~min}$ extension at $72^{\circ} \mathrm{C}$ with a final extension for $5 \mathrm{~min}$ at $72^{\circ} \mathrm{C}$ at the end of 35 cycles. The PCR products were mixed with bromo-phenol blue gel loading dye and were analyzed by electrophoresis on $3.5 \%$ (w/v) agarose gel. The gels were stained in $0.5 \mathrm{mg} / \mathrm{ml}$ ethidium bromide and were documented using gel documentation system (Bio-Rad Laboratories Inc., USA). This was followed by scoring of bands with the help of 50 bp DNA size standard (Fermentas, Lithuania, USA).

\section{Results and Discussion}

\section{Evaluation for grain yield and contributing traits}

The hybrids and inbreds were evaluated for various agronomic and yield related traits (Table 2). The mean values for plant height 
were recorded between 100 and $215 \mathrm{~cm}$ for inbreds CM-128 and KDM-916A, respectively. Among hybrids the plant height ranged from $166 \mathrm{~cm}$ for $\mathrm{H}_{3}$ to $240 \mathrm{~cm} \mathrm{H} 1$.

Hybrid $\mathrm{H}_{1}$ was followed by hybrid $\mathrm{H}_{4}, \mathrm{H}_{2}$ and $\mathrm{H}_{5}$. The inbreds recorded an average plant height of $125.29 \mathrm{~cm}$ against $205.70 \mathrm{~cm}$ for hybrids. The range of variability for days to tassel emergence across a set of inbreds and hybrids was recorded between 59 for KDM$343 \mathrm{~A}$ to $67 \mathrm{KDM}-914 \mathrm{~A}$ DAS. Average of 61.9 and 60.7 DAS were recorded for inbreds and hybrids, respectively. The mean values for days of silk emergence ranged from 61.5 and 67.0 days within inbreds and from 63.5 to 64.5 within hybrids. The early silk emergence was recorded for CM-128 with 61.50 DAS while all the hybrids recorded around 64.0 days to emergence of silk with low CV $(1.40 \%)$. Days to maturity ranged from 126.5 to 136 days among inbreds with inbred KDM$361 \mathrm{~A}$ recording 126.5 days and KDM-916A recording 136.0 days. Hybrid $\mathrm{H}_{2}$ recorded early maturity with 127 DAS while as Hybrid H3 with 137 days recorded maximum days to maturity. Maximum ear length was recorded for inbred KDM-332A with $10.10 \mathrm{~cm}$ while the minimum ear length was recorded for CM-128 with $8.50 \mathrm{~cm}$. Ear length ranged from $14.8 \mathrm{~cm}$ to $18.5 \mathrm{~cm}$ for $\mathrm{H} 2$ and $\mathrm{H} 1$, respectively. Among the inbreds, the range of variability for ears per plant was recorded from $1.0 \mathrm{~cm}$ to $1.70 \mathrm{~cm}$ with the mean of 1.29 $\mathrm{cm}$. KDM-332A and KDM-914A recorded highest number of ears per plant with 1.7 ears each followed by KDM-343A and KDM362A both having 1.20 ears per plant. Hybrids H1 was found to be prolific with 2.10 ears per plant. Inbreds recorded the mean kernel rows per ear of 9.86 versus hybrids with average value of 14.51. Among inbreds, highest test weight of $18 \mathrm{gm}$ was recorded for KDM-914 A. Test-weight ranged from 26.5 to $29.5 \mathrm{gm}$ among hybrids with the mean of the 28.40 $\mathrm{gm}$. The mean grain yield per hectare ranged from 19.71 to $25.60 \mathrm{q} / \mathrm{ha}$ for $\mathrm{CM}-128$ and KDM-362A, respectively. Grain yield in hybrids ranged from 54.16 to $78.84 \mathrm{q} / \mathrm{ha}$ (H1) with a mean of $65.08 \mathrm{q} / \mathrm{ha}$.

\section{Characterization of single cross hybrids for different morphological attributes}

The five single cross hybrids were evaluated for 31 descriptive traits following (DMR, DUS descriptor). It was found that only nine out of 31 traits studied were polymorphic among hybrids and rest of the traits were completely uniform and monomorphic across all the five hybrids. Out of these nine polymorphic traits, only 'Ear: anthocyanin colouration of glumes of cob' had three different classes, while rest eight were grouped in two contrasting phenotypes. The eight polymorphic traits included: Leaf: Altitude of blade, Tassel: Angle between main axis and lateral branches, Tassel: Altitude of lateral branches, Tassel: Length of main axis, Plant ear placement, Leaf: Width of blade, Ear: Length and Ear: color of top of grain. As many as eight out of 31 traits differed between $\mathrm{H} 3$ and $\mathrm{H} 4$ whereas; $\mathrm{H} 1$ and $\mathrm{H} 4$ differed with respect to only three traits (Table 3 and Fig. 1).

\section{Amplification profile of SSR markers across parents and hybrids}

The marker Umc-2383 amplified $135 \mathrm{bp}$ allele in parents KDM-361A and KDM 343A and 150bp allele in rest of five parents (Fig. 2). Allele size of $135 \mathrm{bp}$ allele was scored in hybrids $\mathrm{H}_{1}$ and $\mathrm{H}_{2}$ while 150bp allele was scored in rest of three hybrids.Umc-2100 amplified 100 bp allele in parents KDM 361A and KDM 343A and 125 bp allele in rest of five parents. Fragment size of $100 \mathrm{bp}$ was scored in a single hybrid $\mathrm{H}_{1}$ while other four hybrids record 125 bp allele. The marker Umc-2245 amplified $125 \mathrm{bp}$ in two parents and amplified $100 \mathrm{bp}$ allele in other five. 
Table.1 The list of SSR markers used for identification of polymorphic markers between parents and multi-locus profiling of hybrids

\begin{tabular}{|c|c|c|c|c|}
\hline S No & Marker & Forward primer sequence & Reverse primer sequence & Bin location \\
\hline 1 & Umc2383 & CATAGACGTGCCCCTTGTCATC & CTCGCAACTGCGCTTCTAGATACT & $1.02-1.03$ \\
\hline 2 & Umc1664 & AATTGTTTACTGCGCTGAAACTCC & CCTCTTTGCCTGTACCGTGTATTC & 1.06 \\
\hline 3 & Umc1147 & GAGAAACCATCGACCCTTCCTAAC & TTCCTATGGTACAGTTCTCCCTCG & 1.07 \\
\hline 4 & Umc2100 & AAAGGCATTATGCTCACGTTGATT & TGACGTGCAAACAACCTTCATTAC & 1.12 \\
\hline 5 & Umc2245 & GCCCTGTTATTGGAACAGTTTACG & CGTCGTCTTCGACATGTACTTCAC & 2.01 \\
\hline 6 & Umc1696 & CTAGGGTTTAACCAACGGGGAG & TAAGGAGAGGGTCGATGAACACAT & 2.1 \\
\hline 7 & Umc1823 & AAAGCCTTACTGTTATTAGGCTAGGCA & AGAAAACCAGCCCCAGATGTTC & 2.03 \\
\hline 8 & Umc1026 & TCGTCGTCTCCAATCATACGTG & GCTACACGATACCATGGCGTTT & 2.04 \\
\hline 9 & Umc2372 & ACCCCTTGCGTTCTCTTCTGTT & CACCAGGCGTAGTGAGACAGC & 2.06 \\
\hline 10 & Umc2144 & CCAGCCCCTATCTATTTGCTTGT & GAATACTATATCACGGTCGGTCGG & 2.08 \\
\hline 11 & Umc1594 & GCCAGGGGAGAAATAAAATAAAGC & CACTGCAGGCCACACATACATA & $3.09-3.1$ \\
\hline 12 & Umc2071 & ACTGATGGTGTTCTTGGGTGTTTT & ATACACGCAGTTACCCGAAGGTT & 3.01 \\
\hline 13 & Umc2369 & TTCGTCTGATGAAAGGTTCAGAGG & GATCCTCATCAAGACCAGCAGAGT & $3.02-3.03$ \\
\hline 14 & Umc1644 & CCATAAACTGTTCCTTTGGCACAC & CTTTCACGTGTTAAGGGAGACACC & 3.06 \\
\hline 15 & Bnlg1890 & ACCGGAACAGACGAGCTCTA & GTCCTGCAAAGCAACCTAGC & 4.11 \\
\hline 16 & Bnlg1621 & CTCTTCGATCTTTAAGAGAGAGAGAG & ACACGAGGCACTGGTACTAACG & 4.06 \\
\hline 17 & Umc1478 & GAAGCTTCTCCTCTCGCGTCTC & CAGTCCCAGACCCTAGCTCAGTC & 5.01 \\
\hline 18 & Umc1800 & TTATGGGTGCTGGTGATGTGTATC & GAAAAGCAATCGCTTCTGAGAAAA & 5.05 \\
\hline 19 & Umc2136 & CCAGATGCGGAAGTAGACGG & GATTCGGAGGTGATCTGACCTGT & 5.08 \\
\hline 20 & Umc1766 & ACAAGAAGGAATCGAGAGCAAATG & CTTCGGGATGGAGTCGTAGTTC & 5.01 \\
\hline 21 & Bnlg1306 & CACCTTGAAAGCATCCTCGT & CAAAAACAAATGGCAGCTGA & 5.07 \\
\hline 22 & Umc1918 & CACAAGAACATTATGACGACCGAG & AAGCAGGAGACATCGTTTAAGTCG & 6.03 \\
\hline
\end{tabular}




\begin{tabular}{|c|c|c|c|c|}
\hline 23 & Umc1762 & CTTACTCCAGGCACTCCATACCAT & ATCCAGGTGAATGGTGTTTACGAT & 6.06 \\
\hline 24 & Umc1063 & AGGCCACTGAGCAGGTGAAG & GTGATGGTAGAGGAGTCCTTGGTG & 6.07 \\
\hline 25 & Umc1018 & GAACGGATATTGGAACCTGTGC & GTGCACGGTGTCGTACTTGAAC & 6.01 \\
\hline 26 & Phi452693 & CAAGTGCTCCGAGATCTTCCA & CGCGAACATATTCAGAAGTTTG & 6.04 \\
\hline 27 & Umc1424 & CCGGCTGCAGGGGTAGTAGTAG & ATGGTCAGGGGCTACGAGGAG & 6.06 \\
\hline 28 & Phi129 & GTCGCCATACAAGCAGAAGTCCA & TCCAGGATGGGTGTCTCATAAAACTC & 6.05 \\
\hline 29 & Umc1002 & AGCTAGCTATACACCGCCAGG & TCAGTTTGGAACAGGGAAAAGTA & 6 \\
\hline 30 & Phi051 & GGCGAAAGCGAACGACAACAATCTT & CGACATCGTCAGATTATATTGCAGACCA & 7.05 \\
\hline 31 & Umc1036 & CTGCTGCTCAAGGAGATGGAGA & GACACACATGCACGAGCAGACT & 7.02 \\
\hline 32 & Umc1708 & GATATGTCGAGCTTCGCTGGAG & CGCACACTAAAGCATCCTTAACCT & 7.04 \\
\hline 33 & Umc2392 & CAGAGACCTCGACTTCGACCAC & CTTCTGCTTCTGCTCGACCTTCT & 7.01 \\
\hline 34 & Bnlg1056 & ATCGTTGTTGGGTACACGGT & ACGGGTAGTGGTGAAGATGC & 8.08 \\
\hline 35 & Umc1141 & AGAGGAGAAAGAGACAGACAGGCA & CAGGAACTGAATGAAAGCAACTCA & 8.06 \\
\hline 36 & Umc1415 & GTGAGATATATCCCCGCCTTCC & AGACTTCCTGAAGCTCGGTCCTA & 8.04 \\
\hline 37 & Umc1786 & ACCGTGACTTCCTCCTCATAACTG & CATTTTTCGCATTTAGGAAATCCA & 8.01 \\
\hline 38 & Phi067 & CTGCAAAGGTAAGCACTAGGATGCT & CATCATTGATCCGGGTGTCGCTTT & 9.01 \\
\hline 39 & Phi061 & GACGTAAGCCTAGCTCTGCCAT & AAACAAGAACGGCGGTGCTGATTC & 9.03 \\
\hline 40 & Umc1310 & AACTCCGAGATCTACGACAACAGC & GAGGAAGAGTTGGCCAGGATG & 9.06 \\
\hline 41 & Umc1675 & GTTCTTCCTCTTCCCCATCAGTCT & ATAGCTGCGCGTAAAGCAACC & $9.07-08$ \\
\hline 42 & Umc1640 & ACTACACGGTGTGAGATGTGATCG & GTCGTCGCAAGAACAACAAGG & 10.07 \\
\hline 43 & Umc1077 & CAGCCACAGTGAGGCACATC & CAGAGACTCTCCATTATCCСТCCA & 10.04 \\
\hline 44 & Mmc0501 & TGCTGAACACTCTAAGCAATAC & ATTACTCTACTCGCTGCCTG & 10.02 \\
\hline 45 & Bmc1655 & ATTAAAATCTTGCTGATGGCG & TTCTGTTCCCGCCTGTACTT & 10.03 \\
\hline
\end{tabular}


Table.2 Evaluation of parental inbreds and hybrids for yield and yield contributing traits

\begin{tabular}{|c|c|c|c|c|c|c|c|c|c|c|c|}
\hline Entry & PH & DT & DS & DM & EL & EP & KE & KR & ED & SW & GY \\
\hline KDM-361A & 102.3 & 60.3 & 63.5 & 126.1 & 8.8 & 1.1 & 9.2 & 15.4 & 3.1 & 14.5 & 20.1 \\
\hline KDM-343A & 108.5 & 59.4 & 63.3 & 130.4 & 9.7 & 1.2 & 11.5 & 12.5 & 3.5 & 15 & 20.6 \\
\hline KDM-332A & 102.7 & 63.3 & 64.6 & 133.4 & 10 & 1.7 & 9.2 & 16.5 & 2.8 & 17.2 & 25.2 \\
\hline KDM-914A & 128.6 & 67.3 & 67.7 & 131.2 & 9.4 & 1.7 & 11.5 & 12.9 & 3.1 & 18.8 & 25.5 \\
\hline KDM-362A & 120.4 & 64.6 & 65 & 131.8 & 9.7 & 1.2 & 10.1 & 16 & 2.3 & 16.1 & 25.6 \\
\hline KDM-916A & 215.3 & 60.6 & 63.5 & 136.3 & 9.6 & 1.1 & 11.8 & 12.5 & 2.8 & 14.4 & 19.9 \\
\hline CM-128 & 100.3 & 59.4 & 61.1 & 135.3 & 8.5 & 1.0 & 8.8 & 15.4 & 2.3 & 16.3 & 19.7 \\
\hline $\begin{array}{l}H_{1}(\text { KDM-361A X } \\
\text { KDM-343A) }\end{array}$ & 240.3 & 59.5 & 63.2 & 131.4 & 18.5 & 2.1 & 16.3 & 40.8 & 5.5 & 29.5 & $\mathbf{7 8 . 8}$ \\
\hline $\begin{array}{l}\mathrm{H}_{2}(\mathrm{KDM}-332 \mathrm{~A} X \\
\mathrm{CM}-128)\end{array}$ & 206.3 & 59.3 & 63.7 & 127.1 & 14.8 & 1.5 & 13.5 & 34 & 3.8 & 29.4 & 54.1 \\
\hline $\begin{array}{l}\mathrm{H}_{3}(\mathrm{KDM}-914 \mathrm{~A} X \\
\text { KDM-362A })\end{array}$ & 166.4 & 60.4 & 64.2 & 137.7 & 17.3 & 1.9 & 15.0 & 40.5 & 4.8 & 29.4 & 70.4 \\
\hline $\begin{array}{l}\mathrm{H}_{4}(\mathrm{KDM}-916 \mathrm{~A} X \\
\mathrm{CM}-128)\end{array}$ & 227.5 & 61.8 & 63.1 & 129.1 & 15.6 & 2.0 & 13.2 & 40.9 & 5.2 & 26.5 & 57.4 \\
\hline $\begin{array}{l}\mathrm{H}_{5}(\mathrm{KDM}-332 \mathrm{~A} X \\
\text { KDM-916A) }\end{array}$ & 188.0 & 63.4 & 64.2 & 132.3 & 17.4 & 1.7 & 14.4 & 40.7 & 4.0 & 27.7 & 64.4 \\
\hline Mean & 158.9 & 61.6 & 63.9 & 131.8 & 12.4 & 1.5 & 12.0 & 24.8 & 3.6 & 21.2 & 40.1 \\
\hline Sd & 54.3 & 2.5 & 1.5 & 3.5 & 3.9 & 0.4 & 2.5 & 13.0 & 1.1 & 6.6 & 22.9 \\
\hline CV (\%) & 34.2 & 4.1 & 2.4 & 2.7 & 31.4 & 25.4 & 20.5 & 52.4 & 30.2 & 30.9 & 57.0 \\
\hline Min & 100.3 & 59.3 & 61.1 & 126.1 & 8.5 & 1.0 & 8.8 & 12.5 & 2.3 & 14.4 & 19.7 \\
\hline Max & 240.3 & 67.3 & 67.7 & 137.7 & 18.5 & 2.1 & 16.3 & 40.9 & 5.5 & 29.5 & 78.8 \\
\hline Mean Inbreds & 215.3 & 67.3 & 67.7 & 136.3 & 10 & 1.7 & 11.8 & 16.5 & 3.5 & 18.8 & 25.6 \\
\hline Mean Hybrids & 240.3 & 63.4 & 64.2 & 137.7 & 18.5 & 2.1 & 16.3 & 40.9 & 5.5 & 29.5 & 78.8 \\
\hline
\end{tabular}

PH: Plant height (cm); DT: Days to tassel emergence; DS: Days to silk emergence; DM: Days to maturity; EL: Ear length (cm); EP: Ears per plant; KE: Kernel rows per ear; KR: Kernels per row; ED: Ear diameter (cm); SW: 100seed weight (g); GY: Grain yield (q/ha) 
Table.3 Characterization of single cross hybrids for different morphological attributes

\begin{tabular}{|c|c|c|c|c|c|c|}
\hline \begin{tabular}{|l|l|} 
S \\
No.
\end{tabular} & Character & $\begin{array}{l}\text { KDM-361A } \\
\text { KDM-343A }\end{array}$ & $\begin{array}{l}\text { KDM-332A X } \\
\text { CM-128 }\end{array}$ & $\begin{array}{l}\text { KDM-914A X } \\
\text { KDM-362A } \\
\end{array}$ & $\begin{array}{l}\text { KDM-916A X } \\
\text { CM-128 } \\
\end{array}$ & \begin{tabular}{|l|} 
KDM-332A X \\
KDM-916A \\
\end{tabular} \\
\hline 1 & $\begin{array}{l}\text { Leaf angle between blade and stem (on } \\
\text { leaf just above upper ear) }\end{array}$ & Small & Small & Small & Small & Small \\
\hline 2 & $\begin{array}{l}\text { Leaf : Altitude of blade (on leaf just } \\
\text { above upper ear) }\end{array}$ & Straight & Straight & Drooping & Straight & Drooping \\
\hline 3 & $\begin{array}{l}\text { Stem : Anthocyanin colouration of } \\
\text { brace roots }\end{array}$ & Absent & Absent & Absent & Absent & Absent \\
\hline 4 & Tassel : Time of anthesis & Early & Early & Early & Early & Early \\
\hline 5 & $\begin{array}{l}\text { Tassel : Anthocuanin colouration of } \\
\text { base of glume }\end{array}$ & Absent & Absent & Absent & Absent & Absent \\
\hline 6 & $\begin{array}{l}\text { Tassel : Anthocyanin colouration of } \\
\text { glumes excluding base }\end{array}$ & Absent & Absent & Absent & Absent & Absent \\
\hline 7 & $\begin{array}{l}\text { Tassel : Anthocyanin colouration of } \\
\text { anthers (in middle third of main axis } \\
\text { on fresh anthers) }\end{array}$ & Absent & Absent & Absent & Absent & Absent \\
\hline 8 & $\begin{array}{l}\text { Tassel : Density of spikelets (in middle } \\
\text { third of main axis) }\end{array}$ & Sparse & Sparse & Sparse & Sparse & Sparse \\
\hline 9 & $\begin{array}{l}\text { Tassel : Angle between main axis and } \\
\text { lateral branches (in lower third of } \\
\text { tassel) }\end{array}$ & Wide & Narrow & Narrow & Wide & Narrow \\
\hline 10 & $\begin{array}{l}\text { Tassel : Altitude of lateral branches (in } \\
\text { lower third of tassel) }\end{array}$ & Curved & Straight & Straight & Curved & Straight \\
\hline 11 & Ear : Time of silk emergence & Early & Early & Early & Early & Early \\
\hline 12 & $\begin{array}{l}\text { Ear : Anthocyanin colouration of silks } \\
\text { (on day of emergence) }\end{array}$ & Absent & Absent & Absent & Absent & Absent \\
\hline 13 & $\begin{array}{l}\text { Leaf Anthocyanin colouration of } \\
\text { sheath (below the ear) }\end{array}$ & Absent & Absent & Absent & Absent & Absent \\
\hline 14 & $\begin{array}{l}\text { Tassel : Length of main axis above } \\
\text { lowest side branch) }\end{array}$ & Medium & Medium & Medium & Long & Long \\
\hline 15 & Plant ear placement & Medium & Low & Medium & Low & Low \\
\hline 16 & $\begin{array}{l}\text { Leaf : Width of blade (leaf of upper } \\
\text { ear) }\end{array}$ & Broad & Medium & Broad & Broad & Medium \\
\hline 17 & Ear: Length without husk & Long & Long & Long & Medium & Long \\
\hline 18 & Ear. Diameter without husk (in middle) & Large & Large & Large & Large & Large \\
\hline 19 & Ear : Shape & Conical & Conical & Conical & Conical & Conical \\
\hline 20 & Ear: Number of rows of grains & Many & Many & Medium & Many & Many \\
\hline 22 & $\begin{array}{l}\text { Ear : Type of grain (in middle third of } \\
\text { ear) }\end{array}$ & Flint & Flint & Flint & Flint & Flint \\
\hline 23 & Ear: color of top of grain & Yellow & Yellow with cap & Yellow with cap & Yellow & Yellow \\
\hline 24 & $\begin{array}{l}\text { Ear : anthocyanin colouration of } \\
\text { glumes of cob }\end{array}$ & White & Dark purple & Light purple & White & Light purple \\
\hline 25 & $\begin{array}{l}\text { Kernel : row arrangement (middle of } \\
\text { ear) }\end{array}$ & Straight & Straight & Straight & Straight & Straight \\
\hline 26 & Kernel : poppiness & Absent & Absent & Absent & Absent & Absent \\
\hline 27 & Kernel : sweetness & Absent & Absent & Absent & Absent & Absent \\
\hline 28 & Kernel : waxiness & Absent & Absent & Absent & Absent & Absent \\
\hline 29 & Kernel : apaqueness & Absent & Absent & Absent & Absent & Absent \\
\hline 30 & Kernel : Shape & Toothed & Toothed & Toothed & Toothed & Toothed \\
\hline 31 & Kernel : 1000 kernel & Medium & Medium & Medium & Medium & Medium \\
\hline
\end{tabular}


Table.4 Simple sequence repeat marker based profile of five single cross hybrids

\begin{tabular}{|c|c|c|c|c|c|c|}
\hline$\sum_{n}^{0}$ & 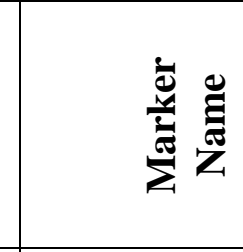 & 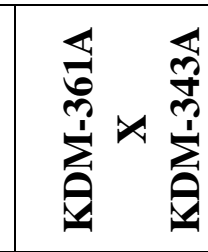 & 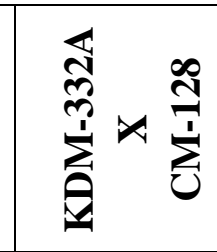 & $\frac{\mathbb{s}}{a}$ & 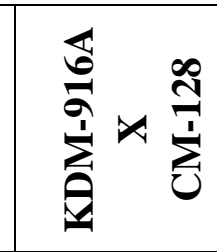 & 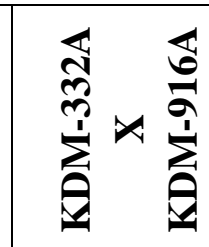 \\
\hline 1 & Umc-2383 & 135 & 150 & 150 & 135 & 150 \\
\hline 2 & Umc-1664 & $140 / 120$ & $140 / 120$ & $140 / 120$ & $140 / 120$ & $140 / 120$ \\
\hline 3 & Umc-1147 & $100 / 85$ & $120 / 85$ & 85 & $120 / 85$ & 85 \\
\hline 4 & Umc-2100 & 100 & 125 & 125 & 125 & 125 \\
\hline 5 & Umc-2245 & 100 & 100 & 125 & 100 & 100 \\
\hline 6 & Umc-1696 & NA & 150 & 150 & 150 & 150 \\
\hline 7 & Umc-1823 & 100 & 100 & 100 & 100 & 100 \\
\hline 8 & Umc-1026 & 150 & 150 & 150 & 150 & 150 \\
\hline 9 & Umc-2372 & $150 / 120$ & $140 / 120$ & $150 / 120$ & $140 / 120$ & $150 / 120$ \\
\hline 11 & Umc-1594 & $140 / 100$ & 140 & 140 & 140 & 140 \\
\hline 12 & Umc-2071 & 150 & 150 & 150 & 150 & 150 \\
\hline 13 & Umc-2369 & 300 & 300 & 300 & 300 & 300 \\
\hline 16 & Bnlg-1621 & 200 & 200 & 200 & 200 & 200 \\
\hline 17 & Umc-1478 & 150 & 150 & 150 & 150 & 150 \\
\hline 18 & Umc- 1800 & 150 & 150 & 150 & 150 & 150 \\
\hline 19 & Umc-2136 & 150 & 150 & 150 & 150 & 150 \\
\hline 20 & Umc-1766 & 165 & 165 & 165 & 165 & 165 \\
\hline 21 & Bnlg-1306 & 175 & 175 & 175 & 175 & 175 \\
\hline 24 & Umc-1063 & 150 & 150 & 150 & 150 & 150 \\
\hline 26 & Umc-1036 & 175 & 175 & 175 & 175 & 175 \\
\hline 27 & Phi-129 & 100 & 100 & 100 & 100 & 100 \\
\hline 28 & Phi-051 & 140 & 140 & 140 & 140 & 140 \\
\hline 30 & Umc-2392 & 90 & 90 & 90 & 90 & 90 \\
\hline 31 & Umc-1141 & 100 & 100 & 100 & 100 & 100 \\
\hline 33 & Phi-067 & 200 & 200 & 200 & 200 & 200 \\
\hline 37 & Umc-1675 & 150 & 150 & 150 & 150 & 150 \\
\hline 38 & Umc-1640 & 125 & 125 & 125 & 125 & 125 \\
\hline 41 & Phi-061 & $80 / 60$ & $60 / 80$ & $80 / 60$ & $80 / 60$ & $80 / 60$ \\
\hline 42 & Umc-1002 & $160 / 140$ & 140 & $150 / 170$ & 140 & 140 \\
\hline 43 & Umc-1424 & 140 & $140 / 110$ & 140/110 & 140 & 140 \\
\hline 44 & Phi-452693 & 125 & 125 & 125 & 125 & 105 \\
\hline
\end{tabular}

Bold figured markers are highly polymorphic in discriminating the hybrids 
Table.5 The Probability of identity between hybrids based on amplification SSR barcode using ten SSR markers

\begin{tabular}{|c|c|c|c|c|c|}
\hline & $\mathbf{H}_{1}$ & $\mathbf{H}_{2}$ & $\mathbf{H}_{3}$ & $\mathbf{H}_{4}$ & $\mathbf{H}_{5}$ \\
\hline $\mathrm{H}_{1}$ & & $\mathbf{0 . 1 6}$ & $\mathbf{0 . 1 0}$ & $\mathbf{0 . 1 5}$ & $\mathbf{0 . 1 3}$ \\
\hline $\mathrm{H}_{2}$ & & & $\mathbf{0 . 1 5}$ & $\mathbf{0 . 2 1}$ & $\mathbf{0 . 1 8}$ \\
\hline $\mathrm{H}_{3}$ & & & & $\mathbf{0 . 1 3}$ & $\mathbf{0 . 1 4}$ \\
\hline $\mathbf{H}_{4}$ & & & & & $\mathbf{0 . 1 6}$ \\
\hline $\mathbf{H}_{5}$ & & & & & \\
\hline
\end{tabular}

Fig.1 Close view of single cross hybrid

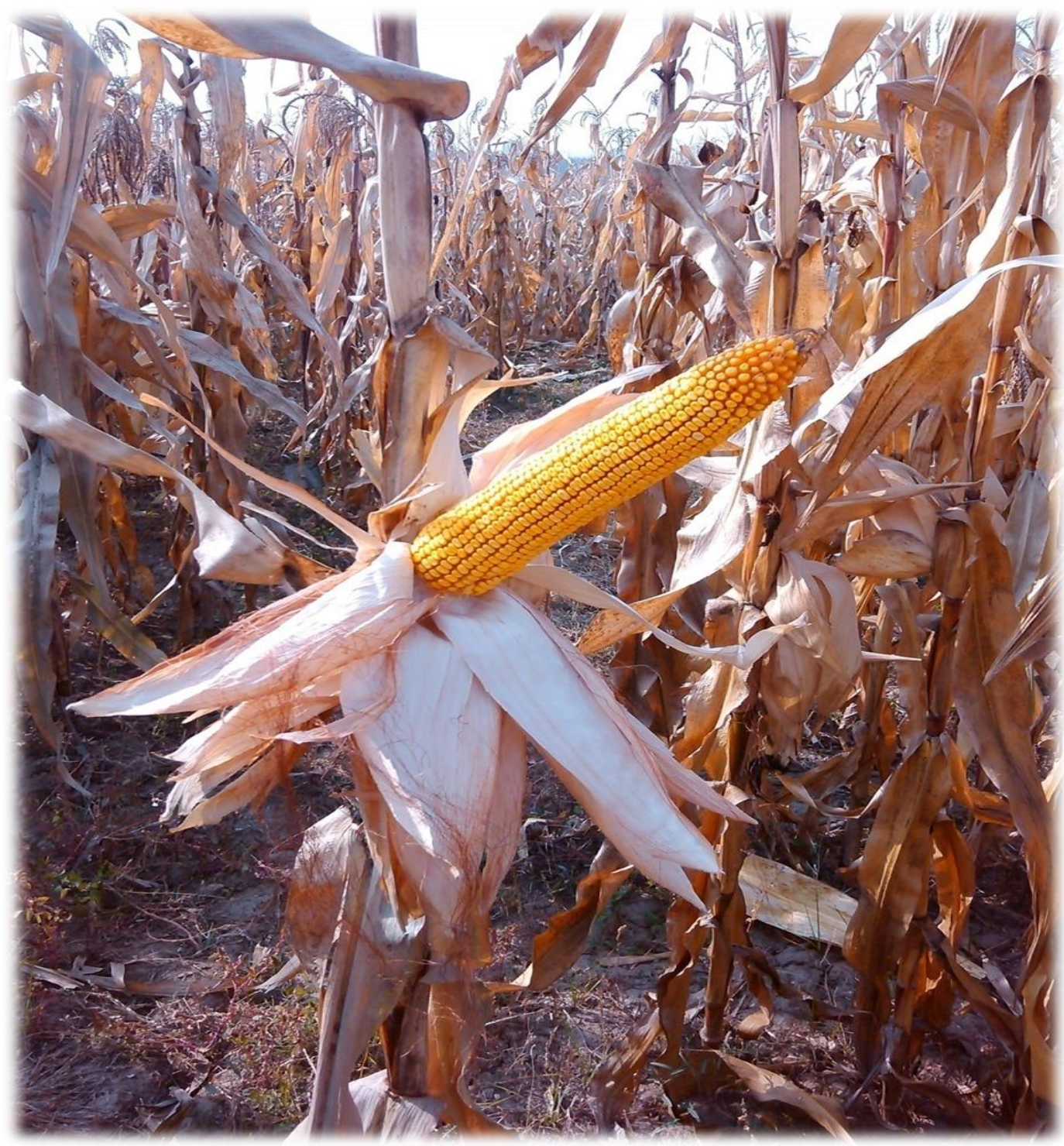


Fig.2 Allelic diversity across five single cross hybrids

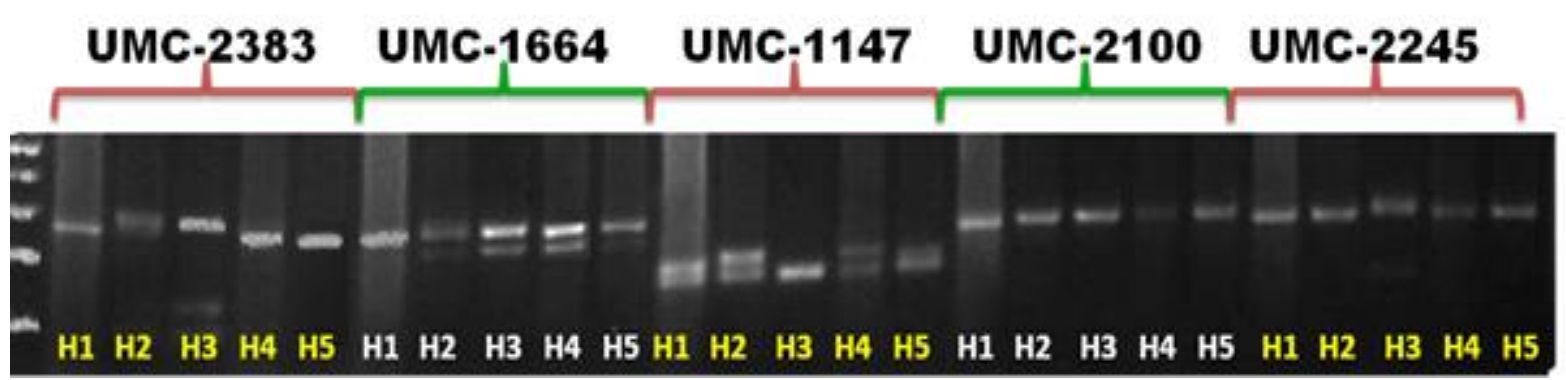

Fig.3 Amplification profile of single cross hybrids against SSR marker UMC-1026 and UMC-2372

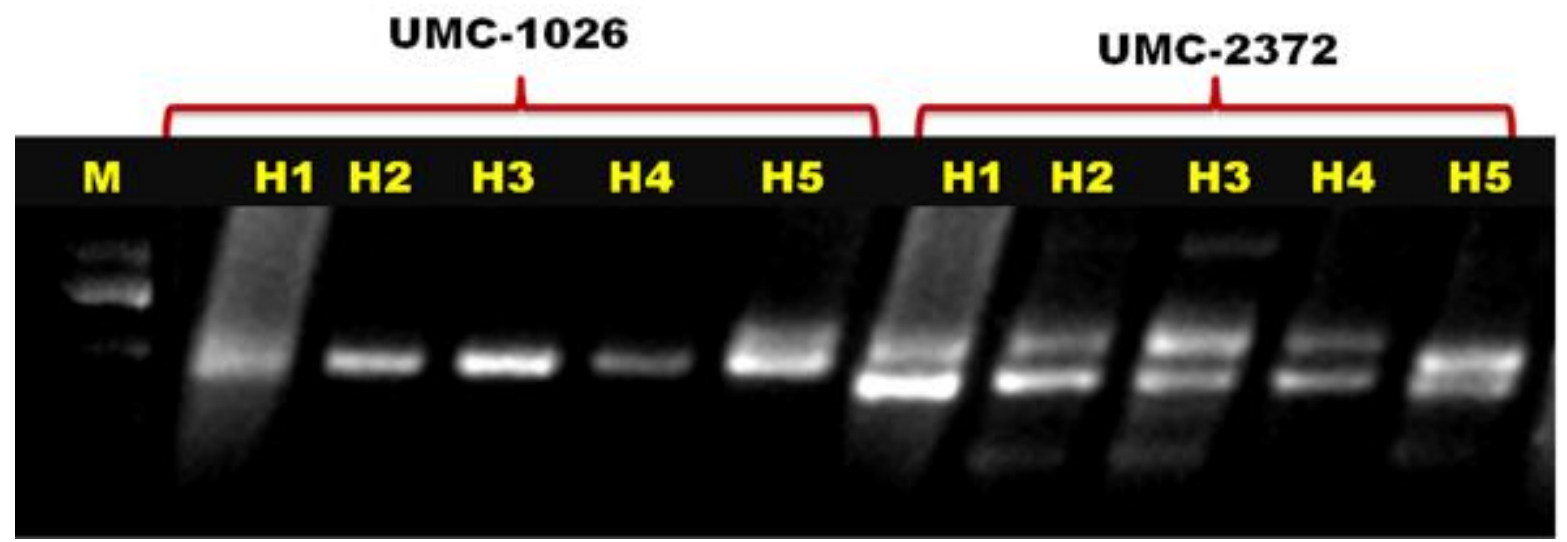

A marker UMC-2372 was polymorphic across the five hybrids.

Designation of single cross hybrids: $\mathrm{H}_{1}$ : KDM-361A X KDM-343A; H2: KDM-332A X CM-128; H3: KDM-914A X KDM-362A; $\mathrm{H}_{4}$ : KDM-916A X CM-128; H : KDM-332A X KDM-916A; M: 50 bp DNA Ladder (MBI, Fermentas, Luthuania)

Allele size of $125 \mathrm{bp}$ was scored in a single hybrid $\mathrm{H}_{3}$ while as rest of 4 hybrids showed 100bp allele.Umc-1696 amplified 150 bp allele in most of the parents and hybrids. Marker Umc 2372 amplified 150 bp allele in parents KDM 361A and KDM 914 A, 140 bp allele in parent CML 128 and in rest four parents 120 bp allele was scored.

In hybrids this marker was polymorphic across all the five hybrids with allele size of 150/120, 140/120,150/120, 140/120, 150/120 bp in hybrids $\mathrm{H}_{1}, \mathrm{H}_{2}, \mathrm{H}_{3}, \mathrm{H}_{4}$ and $\mathrm{H}_{5}$ respectively (Fig. 3). The marker Umc-1708 showed polymorphism between the parents of hybrid $\mathrm{H}_{1}$ with $80 \mathrm{bp}$ and $60 \mathrm{bp}$ alleles whereas; hybrids $\mathrm{H}_{2}$ and $\mathrm{H}_{5}$ amplified 80 bp and $60 \mathrm{bp}$ alleles, respectively. Marker phi 061 was polymorphic across all hybrids with 80 bp (KDM342A, KDM914A, KDM 916A and CM-128) and $60 \mathrm{bp}$ (KDM361A, KDM 332A and KDM 362A) sized alleles (Table 4).

The markers revealed lack of polymorphism across all parents and hybrids with fragment size of $90 \mathrm{bp}$ for the marker Umc-2392; 100 bp for Phi-129, Umc-1141, Bulg-1056, Umc$1823 ; 140 \mathrm{bp}$ for Phi-051; 195 bp for Umc$1036 ; 150$ bp for Umc-1063, Umc-1478, Umc-1675, Umc-1800,Umc-1026 and Umc2136; 165 bp for Umc-1766; 175 bp for Umc- 
1306; 200 bp for Phi-067, Umc-1415, Bnlg $1621 ; 300 \mathrm{bp}$ with respect to markers Umc2071 and Umc-2369.

\section{Fingerprinting of single cross hybrids}

Multi-locus profiling of five single cross hybrids was carried out using 45 genome wide SSR markers. Ten most polymorphic markers were identified based on their PIC values and included the markers, Umc-283, Umc-1664, Umc-1147, Umc-2245, Umc2372, Umc-1594, Phi -061, Umc-1002, Umc1424 and Phi -452693. These ten markers were used to generate DNA profile for five single cross hybrids. SSR Marker Umc-2383 amplified $135 \mathrm{bp}$ allele in hybrids $\mathrm{H}_{1}, \mathrm{H}_{4}$ and 150 bp allele in $\mathrm{H}_{2}, \mathrm{H}_{3}$, and $\mathrm{H}_{5}$. Marker Umc1664 was found to be polymorphic across all the five hybrids amplifying a fragment size of 140 and $120 \mathrm{bp}$ in each of the five hybrids. Umc-1147 showed polymorphism between constitutive parents of hybrids $\mathrm{H}_{1}, \mathrm{H}_{2}$ and $\mathrm{H}_{4}$ and were monomorphic in $\mathrm{H}_{3}$ and $\mathrm{H}_{5} . \mathrm{H}_{1}$ with respect to this marker had an allele size of 100 and $85 \mathrm{bp}$. The marker amplified $120 \mathrm{bp}$ and $85 \mathrm{bp}$ in $\mathrm{H} 2$. The fragment size of 120 and 85 bp was scored in hybrid $\mathrm{H}_{4}$. The marker Umc2245 amplied $100 \mathrm{bp}$ fragment in $\mathrm{H}_{1}, \mathrm{H}_{2}, \mathrm{H}_{4}$ and $\mathrm{H}_{5}$. Umc-2375 was again polymorphic between corresponding parents of all the single cross hybrids. Umc-1594 showed fragment size of $140 \mathrm{bp}$ and $100 \mathrm{bp}$ in $\mathrm{H} 1$ and unique allele of $140 \mathrm{bp}$ in rest four hybrids.

$\mathrm{Ph}-061$ amplified 2 bands each in hybrids $\mathrm{H}_{1}$, $\mathrm{H}_{4}$ and $\mathrm{H}_{5}$ with a size of 80 and $60 \mathrm{bp}$. Hybrid $\mathrm{H}_{1}$ and $\mathrm{H}_{3}$ were characterized having two alleles each against marker Umc-1002. Umc1424 amplified two fragments each in $\mathrm{H}_{2}$ and $\mathrm{H}_{3}$. Phi 452693 amplified 125 bp across all the five hybrids. Taken together each hybrid revealed distinct allele profile across a set of ten markers. Hybrids $\mathrm{H}_{1}$, and $\mathrm{H}_{5}$ recorded 16 and 13 alleles against 10 SSR markers, while $\mathrm{H}_{2}, \mathrm{H}_{3}, \mathrm{H}_{4}$ amplified 14 alleles each.
Based on allelic profile generated for at 10 SSR loci, the hybrids showed maximum genetic divergence of $90 \%$ between $\mathrm{H}_{1}$ and $\mathrm{H}_{3}$. The probability of identity was highest (0.21) between hybrids $\mathrm{H}_{2}$ and $\mathrm{H}_{4}$ and lowest (0.10) between $\mathrm{H}_{1}$ and $\mathrm{H}_{3}$ (Table 5).

During hybrid seed production, greater emphasis is directed towards managing the process to ensure maximum kernel set and high levels of genetic purity (Fonseca et al., 2003). It is necessary to set up a fast, economical and effective system for testing the purity in order to prevent impure seeds from entering the market (Dou et al., 2102).Normally, seed certification for purity and variety distinctness is based on morphological evaluation of seeds and growing plants. For an experimenter Grow out Test is a standard technique to evaluate purity of hybrid seed by comparing seeds and plants in the same stage in identical environmental conditions. These evaluation methods often involve field inspection, which are rigorous, resource and time intensive, prone to error and have very little precision. However, now it has been possible to evaluate the large number of plants at DNA or protein level in a much reproducible manner with greater precision. Among these are use of molecular markers (Perry, 2004; Ali et al., 2008) particularly SSRs (Tautz, 1989) which have proved to be the preferred molecular marker systems for purity identification in some crops (due to their co-dominant nature, high efficiency and simplicity (Wu et al., 2010).

In the present study, the SSR markers Umc2383, Umc-2100, Umc-2245, Umc-1696, Umc-1708 were able to discriminate between parents of one or the other hybrid. The markers Umc-1664, Umc-2372, Phi061revealed polymorphism across parents of all the five hybrids and belonged to chromosomes 1, 2 and 9, respectively. 
Apart from the fact that genetic diversity of maize germplasm is important for planning breeding programmes and conservation of maize germplasm, etc, it is also important that farmers have an opportunity to choose among hybrids the one that will be a choice for highest yields and suitable for environmental stress conditions (Troyer et al., 1983). In Kashmir province, several different hybrids are being pumped by private sector besides few known public bred hybrids those have occupied considerable area. Most of times it becomes difficult for a grower or even seed certification agencies to identify a given hybrid in market merely on the basis of morphological markers or descriptive traits. The hybrids with similar seed characteristics like color, indentation, size and pattern may not easily discern the identity when offered to grower. A set of polymorphic markers in present study was thus used to finger print hybrids. Finally a sub-set of 10 ploymorphic markers were used in order to discriminate five single cross hybrids $\mathrm{H}_{1}, \mathrm{H}_{2}, \mathrm{H}_{3}, \mathrm{H}_{4}$ and $\mathrm{H}_{5}$. Collectively, twelve different alleles (60, 80, 85, 100, 110, 120 125, 135, 140, 150, 160 and $170 \mathrm{bp}$ ) were amplified with the size range of $60 \mathrm{bp}$ to $170 \mathrm{bp}$. Hybrid $\mathrm{H}_{1}, \mathrm{H}_{2}, \mathrm{H}_{3}$, $\mathrm{H}_{4}$ and $\mathrm{H}_{5}$ amplified 16, 14, 14, 14 and 13 alleles, respectively. Based on SSR profile using 10 markers, the hybrids showed distinct molecular profile amongst themselves. Low probability of identity was recorded between $\mathrm{H}_{1}$ and $\mathrm{H}_{3}(0.10)$. Probability that $\mathrm{H}_{2}$ and $\mathrm{H}_{4}$ shared alleles at 10 markers loci was only $21 \%$ that was the highest among all the paired combinations. The SSR profile standardized here holds a promise and could be used as a key to establish genetic identity of hybrids sampled form open market or farmers' custody, with extreme precision and greater reliability.

Protection of Plant varieties and Farmers Right authority insists on characterization and registration of extant, farmers and new varieties as a part of national and botanical asset. Pinnisch et al., (2012) advocated characterizing inbred lines which serve as the seed parents to commercially grown maize hybrids. Therefore, a set of inbreds was characterized using 31 morphological traits following Maize descriptor (DMR, New Delhi). Only nine out of 31 traits studied were polymorphic among hybrids among ear characters only traits 'ear length' and 'grain color' were found polymorphic. As many as eight out of 31 traits differed between $\mathrm{H}_{3}$ and $\mathrm{H}_{4}$ whereas, $\mathrm{H}_{1}$ and $\mathrm{H}_{4}$ differed with respect to only three traits, therefore, revealed high similarity. The marker analysis based on SSRs revealed 4-5 fold more genetic divergence among hybrids when compared to that based on morphological traits. In fact only 10 SSR markers revealed $80-90 \%$ divergence among hybrids that was much higher than what was found based on phenotypic trait variation among the five hybrids. SSRs have been known and several occasions advocated to be markers of choice for estimation traits diversity among hybrids and also for establishing identify and genetic purity of hybrids in crop like maize (Natalya et al., 2002; Wang et al., 2011).

\section{References}

Ali, M.A., et al., 2008. Hybrid authentication in upland cotton through RAPD analysis. Aus. J. of Crop Sci., 2(3), 141149.

Anonymous, 2012. Digest of statistics., Directorate of Economics \& Statistics Planning and Development Department, Govt. of J\&K, Srinagar pp. 94-100.

Anonymous, 2013. Project Director Review, All India Coordinated Annual Maize Workshop, 6-8, April, ANGRAU, Hyderabad.

Asif, M., Rahman M and Zafar Y 2006. Genotyping analysis of six maize (Zea mays L.) hybrids using DNA 
fingerprinting technology. Pak. J. Bot., 38(5): 1425-1430.

Dou, X., Yan M, Xu Y, Hussain K, Liu Y, Lin F. 2012. Identification and purity testing of maize hybrids with one parent in common by ultrathin-layer isoelectric focusing of seed salt-soluble proteins. Turk J Agric For 36: 267-273.

Fonseca, A.E., et al., 2003. Tassel morphology as an indicator of potential pollen production in maize. Crop Management. Online doi, 10.1094/CM2003-0804-01-RS.

Kalinka Carla Padovani de Carvalho Salgado, et al., 2006. Genetic purity certificate in seeds of hybrid maize using molecular markers. Rev. Bras. Sementes, 28(1), Pelotas.

Liu-Jie, Liu-Gongshe, Li-Dianrong, RiDongmei. Wang Hao and Wang-Daojie, 2000. Identification of genetic purity of seed of hybrid rape with RAPD.Chinese J. Appl. Envorn. Biol., 6(6): 526-529.

Mao, C. X., Vermani, S. S., and Kumar, I. 1996. Technological innovations to lower the cost of hybrid rice seed production.Proc, of 3rd Intsymp. On hyb. Rice. pp: 111-123.

Natalya, S., McMullen MD, Schultz L and Edward H. C., 2002. Development and mapping of SSR markers for maize. Plant Molecular Biology 48: 463-481

Pallavi, H. M., Maithreyee. M. N. and Gowda $\mathrm{R}$ 2010. Application of molecular markers in hybrid purity testing.
Summer school on advances in quality seed production, processing and marketing. pp: 220-223.

Perry, D.J., 2004. Identification of Canadian durum wheat varieties using a single PCR. Theor. App. Gen., 109(1), 55-61.

Pinnisch, R., Mowers R, Trumpy H, Walejko $\mathrm{R}$, Bush D 2012. Evaluation of maize (Zea mays L) inbred lines for yield component traits and kernel morphology. Maydica 57:1-5.

Singh, R.K., and Chaudhary, B. D. 1985. Biometrical Methods in Quantitative Genetic Analysis, Kalyani Publishers, New-Delhi. Revd Ed. pp: 39-68.

Srivastava, J., And Jaffee, S. 1993. Best practices for moving seed technology. The World Bank, Washington DC, Technical Paper No 213,

Troyer, A.F., Openshaw S.J. and Knittle K.H. 1983. Measurement of genetic diversity among commercial corn hybrids. Method. In: Agronomy abstracts. ASA, Madison, WI, p: 83.

Wang, F., Tian, H, Zhao J, Yi H, Wang L, Song W. 2011. Development and characterization of a core set of SSR markers for fingerprinting analysis of Chinese maize varieties. Maydica 56: 111.

Wu, M., et al., 2010. Rapid and reliable purity identification of F1 hybrids of Maize (Zea mays L.) using SSR markers. Plant Breeding, 4(3), 381-384.

\section{How to cite this article:}

Bhat, M.I., Gulzaffar, Z.A. Dar, A.A. Lone, A.B. Shikari, G. Ali, M.A. Wani, G.H. Khan, A. Gazal and Lone, R.A. 2017. Genetic Purity Analysis in Maize under Temperate Conditions. Int.J.Curr.Microbiol.App.Sci. 6(9): 2710-2722. doi: https://doi.org/10.20546/ijcmas.2017.609.334 